\title{
Simple Interpretation of the Dynamics of Mutually Coupled Semiconductor Lasers with Detuning
}

\author{
Raúl Vicente $^{a}$, Josep Mulet ${ }^{a}$, Marc Sciamanna $^{b}$ and Claudio R. Mirasso ${ }^{a, c}$ \\ ${ }^{a}$ Departament de Física, Universitat de les Illes Balears, Crta. Valldemossa Km 7.5, E-07122, \\ Palma de Mallorca, Spain; \\ ${ }^{b}$ Faculté Polytechnique de Mons, Service d'Electromagnétisme et de Télécommunications, \\ Boulevard Dolez 31, B-7000, Mons, Belgium; \\ ${ }^{c}$ Electrical Engineering Department, University of California, Los Angeles, Los Angeles, CA, \\ 90095-1594, USA
}

\begin{abstract}
We investigate the synchronization properties of two mutually-coupled semiconductor lasers (SL) in a face to face configuration, when a non-negligible injection delay time is taken into account. Under the appropriate conditions, we derive a thermodynamic potential analog to the one studied by Mork et al. ${ }^{1,2}$ and by Lenstra ${ }^{3}$ for a semiconductor laser subject to an optical feedback. In this context, the role that noise and detuning play in the dynamics of the system is clearly identified. When operating in the Low Frequency Fluctuations (LFF) regime, the effect of the detuning on the leader-laggard operation is also analyzed. Finally, we focus on the short intercavity regime and we study the influence of the detuning and the propagation phase on the dynamics of each laser.
\end{abstract}

Keywords: Semiconductor Lasers, Delay, Instabilities in Diode Lasers, Bidirectional Coupling.

\section{INTRODUCTION}

The nonlinear dynamics and synchronization of mutually coupled oscillators have important applications in fields ranging from the high-power laser arrays ${ }^{4}$ to central issues in neuroscience. ${ }^{5}$ In fact, synchronization was first discovered in two mutually coupled oscillators. ${ }^{6}$ Despite of their inherent interest, only few studies have focused on the instabilities arising from the mutual coupling of two semiconductor lasers. ${ }^{7-12}$ So far, the most studied configuration of mutually interacting laser systems is the evanescent coupling of solid-state lasers. However, due to the fundamental differences between the face-to-face and the evanescent coupling, new phenomena can be expected for the former. Moreover, the excellent controllability of semiconductor lasers in the laboratory make them ideal candidates for the experimental investigation of general phenomena in the theory of mutually coupled oscillators. In this context, besides the frequency and phase locking properties, oscillation death by delay, ${ }^{12}$ localized synchronization ${ }^{7}$ and spontaneous symmetry-breaking ${ }^{8}$ among others effects, has been recently proved to occur in bidirectionally coupled semiconductor laser systems.

There are different options to mutually couple two edge-emitting semiconductor lasers. In the optoelectronic case, ${ }^{12}$ the light coming from one laser facet is converted into electrical current that is added to the bias of the other laser. In the incoherent optical case, ${ }^{13}$ the TE mode of each laser output is 90 degrees rotated before entering into the other laser cavity. It is assumed that in this scheme, the injected fields act only on the carrier population and do not interact with the intracavity lasing fields. Finally, the richer option from the dynamical point of view, and the one in which we focus our attention, is the coherent optical mutual coupling. It represents the mutual injection of the TE mode of each laser into the other laser cavity. In this last case, the parameters controlling the dynamics of both lasers are the coupling strength, delay injection time and propagation phase. These quantities and the detuning between the free-running emission frequencies will be our main bifurcation parameters along this work.

Further author information: (Send correspondence to R.V.)

R.V.: E-mail: raulv@imedea.uib.es, Telephone: 34971172505 
Even in the case that rate equations models are used to describe the dynamics of the system under study, the complexity of the mathematical structure of the set of equations we are dealing with is huge. Consequently, a great advantage has been taken from the consideration of special limits where some approximations are fully justified. On the one hand, one can use the asymptotic analysis motivated from the very different time scales introduced by the photon lifetime, the carrier lifetime and the injection delay time. ${ }^{11,14}$ In particular, Ref.[11] analyzed the case of large distance between the two lasers. On the other hand, it is also common the study of the dynamics of a given system when the perturbation at which is subjected is small, with the hope that the complete understanding of this relatively simple situation will shed light into the interpretation of the more complex dynamics occurring for larger perturbation amplitudes. In this context, phase dynamics models are particularly popular and we will make use of one of them to describe the dynamics in the low coupling limit.

The paper is organized as follows. In Section 2 we present the rate equations describing two spatiallyseparated bidirectionally-coupled semiconductor lasers. Section 3 tackles the derivation of a phase model and the noise role in a potential picture of that model. Section 4 focuses on the role of the detuning in the dynamics of the system for both the short and long coupling delay time limits. Finally, Section 5 summarizes and points out the main conclusions of the paper.

\section{THE MODEL}

We consider our system consisting of two single-mode semiconductor lasers in a face to face configuration, where a moderate amount of coupling, arising from the mutual injection of their transverse electrical fields, is assumed. A rigorous derivation of the model that describes the system under consideration has been already presented in Ref.[9]. From that work, it turns out that the phenomenological model is valid when the coupling strength is weak enough. Since this is our case, we put forward the equations governing the evolution of the electrical fields and carriers in each one of the lasers as

$$
\begin{aligned}
\dot{E}_{1,2} & =\mp i \Delta E_{1,2}+\frac{1}{2}(1+i \alpha)\left[G_{1,2}-\gamma\right] E_{1,2}+\kappa e^{-i \Omega \tau} E_{1,2}(t-\tau)+\sqrt{2 \beta N_{1,2}} \xi_{1,2} \\
\dot{N}_{1,2} & =p \frac{J_{t h}^{s o l}}{e}-\gamma_{e} N_{1,2}-G_{1,2}\left|E_{1,2}\right|^{2} \\
G_{1,2} & \equiv \frac{g\left(N_{1,2}-N_{t}\right)}{1+s\left|E_{1,2}\right|^{2}}
\end{aligned}
$$

where both lasers are taken identical except for a possible detuning between their free-running frequencies $\left(\Delta=\frac{\omega_{2}-\omega_{1}}{2}, \Omega_{0}=\frac{\omega_{2}+\omega_{1}}{2}\right)$. The internal laser parameters used in our numerical simulations correspond to a realistic experimental situation where the linewidth enhancement factor is $\alpha=3.5$, the cavity losses $\gamma=240 \mathrm{~ns}^{-1}$, the carrier decay rate $\gamma_{e}=1.66 \mathrm{~ns}^{-1}$, the differential gain $g=3.2 \times 10^{-6} \mathrm{~ns}^{-1}$, the carrier value at transparency $N_{t}=1.5 \times 10^{8}$, the gain saturation parameter $s=5 \times 10^{-7}$, the spontaneous emission rate $\beta=10^{-5} \mathrm{~ns}^{-1}$ and $e$ is the electron charge. The solitary threshold current is $J_{t h}^{s o l}=60 \mathrm{~mA}$ and $p$ is the injection current with respect to threshold. The rest of parameters will be varied through this paper and their particular values will be specified in each section.

Neglecting the Langevin noise sources in $(1)$, the steady-states $\left(E_{1,2}(t)=\sqrt{P_{1,2}(t)} e^{i \varphi_{1,2}(t)} ; \dot{P}_{1}=\dot{P}_{2}=0\right.$, $\left.\dot{N}_{1}=\dot{N}_{2}=0, \varphi_{1}(t)=\Omega t, \varphi_{2}(t)=\Omega t+\phi\right)$ of the former six-dimensional set of equations can be found systematically with the algorithm presented in Appendix A. In absence of detuning, three types of fixed points can be distinguished: the in-phase symmetric $(\phi=0)$, anti-phase symmetric $(\phi=\pi)$ and asymmetric solutions $(\phi \neq 0, \pi)$. The structure and stability of these stationary solutions have been evaluated for the long distance limit and zero-detuning case in Ref.[11]. Figure 1a shows how these fixed points are typically arranged in the Inversion-Frequency plane $\left(N-N_{t h}\right.$ vs $\Omega \tau$ ) while Figure 1b illustrates how they bifurcate as increasing the coupling strength. Generically, after the saddle-node creation of the symmetric modes, the nodes loss their stability through a Hopf bifurcation while the asymmetric modes emerge and disappear from the symmetric solutions. As in the Lang-Kobayashi model for the case of optical feedback, ${ }^{15}$ for fixed $\kappa$, the symmetric solutions lie on an ellipse with a major semiaxis $C=\kappa \tau \sqrt{1+\alpha^{2}}$. On the other hand, islands of asymmetric 

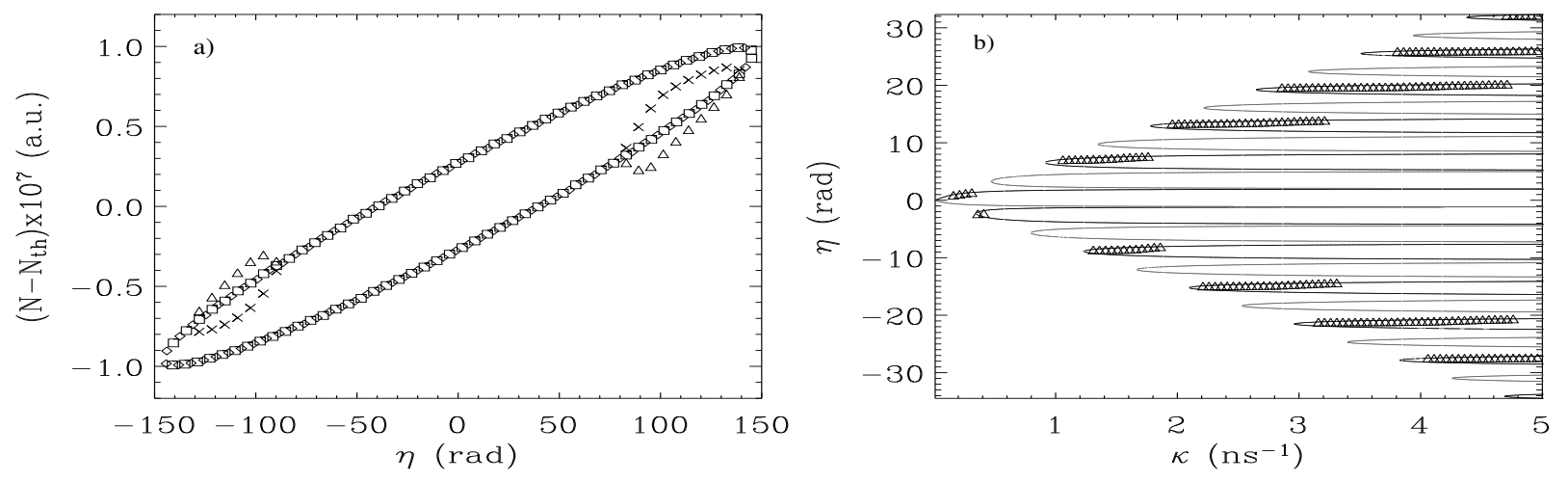

Figure 1. a) Steady-states of eqs. (1)-(2) in the Inversion-Frequency plane for $\kappa=20 \mathrm{~ns}^{-1}$. Squares and diamonds stand for in-phase and anti-phase solutions while crosses (triangles) represent asymmetric modes with positive (negative) phase difference $\phi$. b) Evolution of the modes frequency as increasing the coupling level. Black (grey) solid lines indicate the in-phase (anti-phase) fixed points continuation while the triangles mark the asymmetric modes. In both cases, the pump level and the injection delay time have been set to $p=1$ and $\tau=2 \mathrm{~ns}$.

solutions appear around the edges of the ellipse of symmetric states. However, it is worth mentioning that the number of asymmetric modes may change with the bias current whereas the symmetric ones remain unchanged. The modification of these structures under the presence of detuning or when decreasing the distance between lasers until entering in the short intercavity regime, will have important consequences on the dynamics of the system and they will be studied in Section 4.

The next section is devoted to investigate a reduced model based on the phase equations derived from eqs. (1-2), which we will see remains valid in the low coupling limit. From there, the effect of the spontaneous emission noise and locking regime borders will be predicted.

\section{PHASE DYNAMICS}

For a sufficiently weak coupling, where the relaxation oscillations are still damped, we can assume a constant light intensity and identical in both lasers by neglecting amplitude fluctuations $\left(E_{1,2}(t)=\sqrt{P_{s}} e^{i \varphi_{1,2}(t)}\right)$. This approximation leads to the following coupled equations for the optical phases

$$
\dot{\varphi}_{1,2}(t)=\mp \Delta-\kappa \sqrt{1+\alpha^{2}} \sin \left(\varphi_{1,2}(t)-\varphi_{2,1}(t-\tau)+\Omega \tau+\arctan \alpha\right)+F_{\varphi_{1,2}}(t),
$$

where the $F_{\varphi_{1,2}}(t)$ is the Langevin noise source associated to the each phase. Despite the important reduction that has been achieved, it still remains the difficulty of dealing with a system of delay differential equations (DDE). The next step is to assume a slow variation of the phases over a entire delay time by expanding $\varphi_{1,2}(t) \approx$ $\varphi_{1,2}(t-\tau)+\tau \dot{\varphi}_{1,2}(t-\tau)$. It is worth noting that for DDE these kind of expansions may not lead to valid results. Surprisingly, for small delay $\tau$ the discarded term behaves like $1 / \tau$ if the approximation is taken up to second order or beyond. ${ }^{16}$ Under these conditions, the resulting equations read ${ }^{17}$

$$
\begin{aligned}
& \dot{\varphi}_{1,2}=\mp \Delta-\kappa \sqrt{1+\alpha^{2}} \sin \left(\varphi_{1,2}-\vartheta_{2,1}+\Phi\right)+F_{\varphi_{1,2}}(t), \\
& \dot{\vartheta}_{1,2}=\frac{1}{\tau}\left(\varphi_{1,2}-\vartheta_{1,2}\right)
\end{aligned}
$$




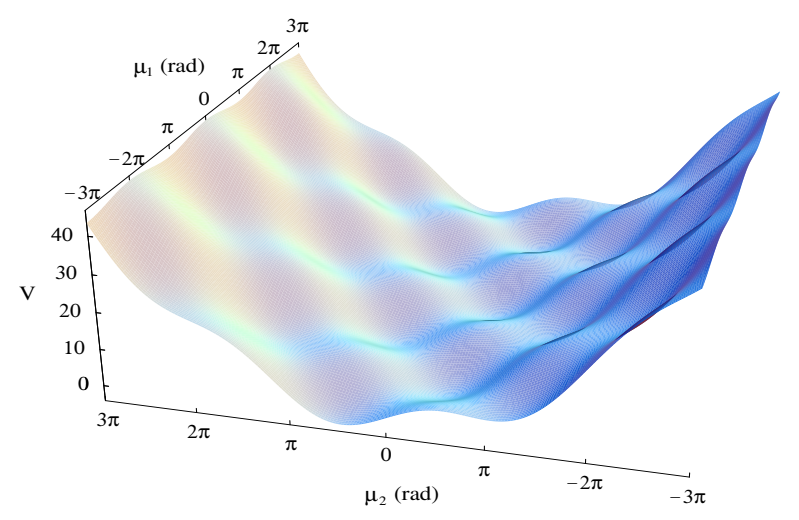

Figure 2. Potential function $V\left(\mu_{1}, \mu_{2}\right)$ computed for the following parameters: $\Delta=0, \tau=2 \mathrm{~ns}, \kappa=0.5 \mathrm{~ns}^{-1}$ and $\Phi=4.43$.

where $\vartheta_{1,2}(t) \equiv \varphi_{1,2}(t-\tau)$ and $\Phi \equiv \Omega \tau+\arctan \alpha \bmod 2 \pi$. Now, defining $\mu_{1,2} \equiv \frac{1}{2}\left[\left(\varphi_{1}-\vartheta_{2}\right) \mp\left(\varphi_{2}-\vartheta_{1}\right)\right]$, i.e. the addition and difference of the injected phases, it is obtained that for these variables there exists a potential function $V$ in terms of which the dynamics is written as

$$
\begin{aligned}
\dot{\mu}_{1,2} & =-\frac{1}{\tau} \frac{\partial V\left(\mu_{1}, \mu_{2}\right)}{\partial \mu_{1,2}}+F_{\mu_{1,2}}(t) \\
V\left(\mu_{1}, \mu_{2}\right) & =\tau \Delta \mu_{1}+\frac{1}{2} \mu_{2}^{2}-C \cos \mu_{1} \cos \left(\Phi+\mu_{2}\right) .
\end{aligned}
$$

Figure 2 shows an example of the potential landscape where the relaxational gradient dynamics take place. Before continuing the analysis, it is worth mentioning that in the steady state operation (i.e. in a potential minima) the variable $\mu_{1}$ represents the phase difference $\phi$ between both lasers while $\mu_{2} / \tau$ accounts for the locked frequency $\Omega$.

At this point, we numerically check the validity of our approximations by comparing the results obtained for the phase difference and frequency in the absence of noise, with the full model [eqs. (1)-(2)] and with the reduced model [eqs. (6)-(7)]. For the same parameters used in the Figure 2, the computation of these quantities with the full model provides, when the initial condition is in the neighborhood a steady-state, the values $\mu_{1}=0$, $\mu_{2}=-1.009812$ while the model based on the potential description gives $\mu_{1}=0, \mu_{2}=-1.009803$. It is then clear that the potential description is valid providing that the coupling values are small enough.

In absence of detuning, it is easy to demonstrate that the potential minima are generically located at coordinates where the $\mu_{1}$ variable equals $n \pi$ with $n \in \mathbb{Z}$. Consequently, the absolute minimum of the potential at which the dynamics of the system approach in the long-time limit can be an in-phase or an anti-phase mode, its selection determined by $\Phi$. In the presence of spontaneous emission noise, the interpretation of these steady-states is associated with the maxima of the probability density function given by the corresponding Fokker-Planck equation. The possible noise-induced mode hopping is illustrated in Figure 3. As consequence of the potential structure, each mode hopping or frequency slip is simultaneously accompanied by a phase slip. The correspondence of the mode hopping with the features of the potential landscape is clearly observed in the bottom panel of Figure 3 , where the stochastic slips occur mainly through the saddles between different minima.

Although computation of Mean First Passage Time (MFPT) between potential wells is much more complicated to perform in several dimensions than in the simple one-dimensional case, ${ }^{18}$ there is still the inversely exponential dependence on the barrier height. Consequently, any increase of $\kappa, \tau$ or $\alpha$ maintained within the margins of validity of the approach here used will lead to the stabilization of the dynamics against the noise.

The role of the detuning on the potential is to break the periodicity in the $\mu_{1}$ variable and to tilt the potential landscape. No in-phase or anti-phase states exist anymore, although for small positive (negative) detuning values 

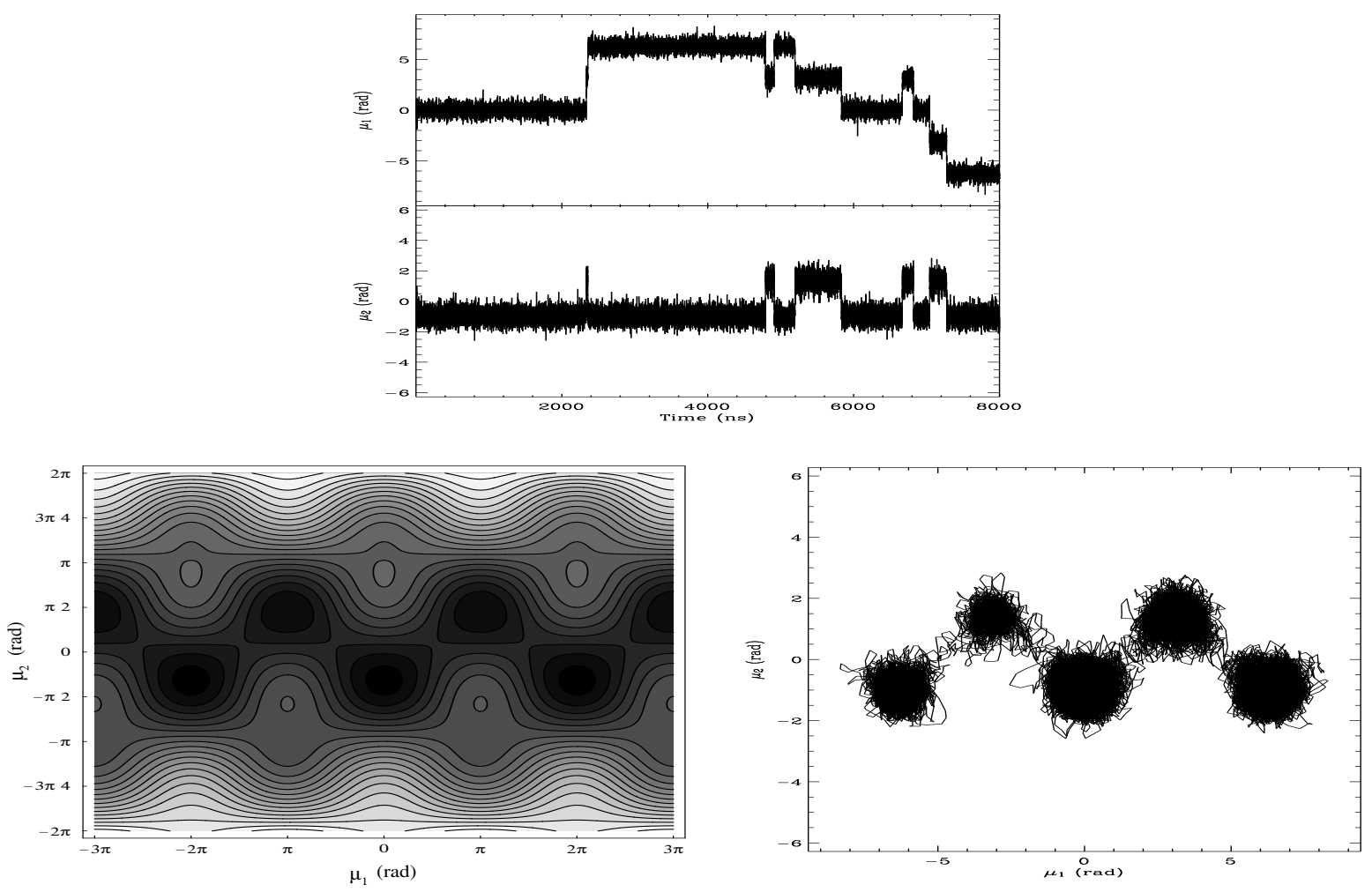

Figure 3. Top panel: Evolution of the phase difference and frequency. Bottom panel: Left graphic is the contour plot of the potential function. Right graphic contains the numerically simulated stochastic path followed by the system in the $\mu_{1}-\mu_{2}$ plane. The parameters used are the same than those corresponding to Figure 2.

infinitely many minima with deeper wells are arranged in the direction of increasing (decreasing) phase difference. Then, in the absence of noise, both lasers would lock to a frequency and phase corresponding to one of these local minima. However, the presence of Gaussian noise (or any other unbounded noise) will make the system to continously jump to deeper minima. If the detuning parameter exceeds a critical value, the potential becomes so tilted that it is unable to produce local minima. In this situation, both lasers cannot manage to lock and no steady-state can be reached. The border of this transition from locking to unlocking regime can be predicted by demanding the condition that the potential function presents a non-zero number of critical points. Eliminating $\mu_{1}$ from the system of equations $\partial V / \partial \mu_{1}=\partial V / \partial \mu_{2}=0$, it is obtained that $\mu_{2}$ has to meet the following condition

$$
\mu_{2}+C \tan \left(\mu_{2}+\Phi\right) \sqrt{\cos ^{2}\left(\mu_{2}+\Phi\right)-\frac{\tau^{2} \Delta^{2}}{C^{2}}}=0
$$

Consequently, in the $\kappa-\Delta$ plane, the Arnold tongue or locking regime area is determined by the region where real solutions for equation (8) exist. An important observation is the fact that changing the value of $\Phi$, the size of the corresponding Arnold Tongue may suffer important modifications and that this effect occurs for both short and long injection delay times. For instance, in Figure 4, the locking limits for values of $\Phi=0, \pi / 4, \pi / 2$ and $3 \pi / 4$ are represented.

Finally, it is worth to recall that the maximum coupling strength for which the former approach is valid (i.e. the maximum coupling for which the relaxation oscillations are still damped), is strongly dependent on the bias current level. Hence, if for $p=1.1$ ( $10 \%$ above solitary threshold) $\kappa_{\max } \approx 3 \mathrm{~ns}^{-1}$, for $p=1.5$ (50\% above solitary threshold) $\kappa_{\max } \approx 9 \mathrm{~ns}^{-1}$ what represents a moderate amount of coupling level. 


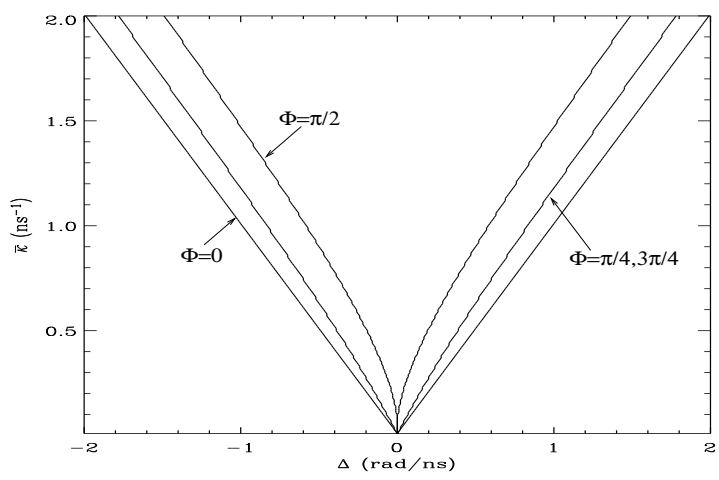

Figure 4. Synchronization regions or Arnold tongues for different values of the injection phase $\Phi$. The modified coupling coefficient that appears in the vertical axis is defined as $\bar{\kappa} \equiv \kappa \sqrt{1+\alpha^{2}}$. The maximum locking size is achieved for $\Phi=n \pi$ while the minimum one is obtained for $\Phi=(2 n+1) \pi / 2, n \in \mathbb{Z}$.

\section{DETUNING EFFECT ON THE DYNAMICS: THE SHORT AND LONG COUPLING DELAY TIME LIMITS}

The detuning influence on the dynamics of unidirectionally coupled laser schemes has been extensively studied in the literature. ${ }^{19}$ In these works, the locking properties and their stability as well as multi-wave mixing processes in the non-locking area have been reported and analyzed. Chirp reduction and bandwidth increment are among the practical applications that benefited from the study of these Master-Slave (M-S) semiconductor lasers systems. However, the fundamental differences between an unidirectional and a mutually coupled scheme make clear the necessity of a separated study of the detuning effect on the dynamics of the system under consideration. Besides the mutual frequency pulling effects, there is the novelty that the propagation phase $\varphi_{0}$ between the lasers may act as an important parameter, unlike in the M-S case where it could be removed from the equations by a simple rescaling of the time variable.

First, we focus on the short intercavity regime by taking the distance between lasers $L=6 \mathrm{~cm}(\tau=0.2 \mathrm{~ns})$. The coupling strength has been chosen to be small $\left(\kappa=5 \mathrm{~ns}^{-1}\right)$, the pumping has been set close to the solitary threshold $p=1$ and the propagation phase is fixed to $\varphi_{0}=0$. Figure 5 (left panel) displays the bifurcation diagram of the optical power of both lasers when the detuning parameter is continuously changed from 0 to 12 GHz. From the diagram, it is observed that for small detuning $(\Delta \nu<0.5 \mathrm{GHz})$ the lasers are still operating in the steady-state condition with a very similar optical power output, until they enter into a period-1 window $(0.5 \mathrm{GHz}<\Delta \nu<1.1 \mathrm{GHz})$. In this regime, the optical power undergoes oscillations in anti-phase dynamics. After this regime, the dynamics enters into a more complex oscillatory regime $(1.1 \mathrm{GHz}<\Delta \nu<1.5 \mathrm{GHz})$, before reaching a sequence of alternating periodic and constant states. For large detunings, it is observed that in the periodic windows, the relative phase between the optical powers depends specifically on the value of the detuning. The right panel of Figure 5 shows the optical spectra of both lasers for selected values of the detuning. Locking, non-locking states and multi-wave mixing phenomena are clearly identified from these graphics. For instance, graphics a) and e) are examples of the locking of both lasers to the most negative mode, while graphics d) and f) show a clear behavior of non-locking states. Interestingly, it is noticed that even when the system is unable to lock for $\Delta \nu=4 \mathrm{GHz}$, it is able to do so for the larger value of the detuning $\Delta \nu=6 \mathrm{GHz}$, although for larger values of the frequency mismatch it is again in an unlocked state. In these unlocked states for large detuning, the periodic oscillations of each laser have a frequency very similar to the detuning value $\Delta \nu$.

Steady-states and typical trajectories, after transients have died out, are plotted in Figure 6 for the same parameters as those used in the right panel of Figure 5. The phase space used to visualize the dynamics is the Inversion-Frequency plane. The description of each graphics is as follows: a) both lasers lock to the most negative frequency mode, which is stable for small values of the detuning, b) each laser is operating in different limit cycles located at different frequencies ranges, c) there is an increment of the complexity of the trajectory, 

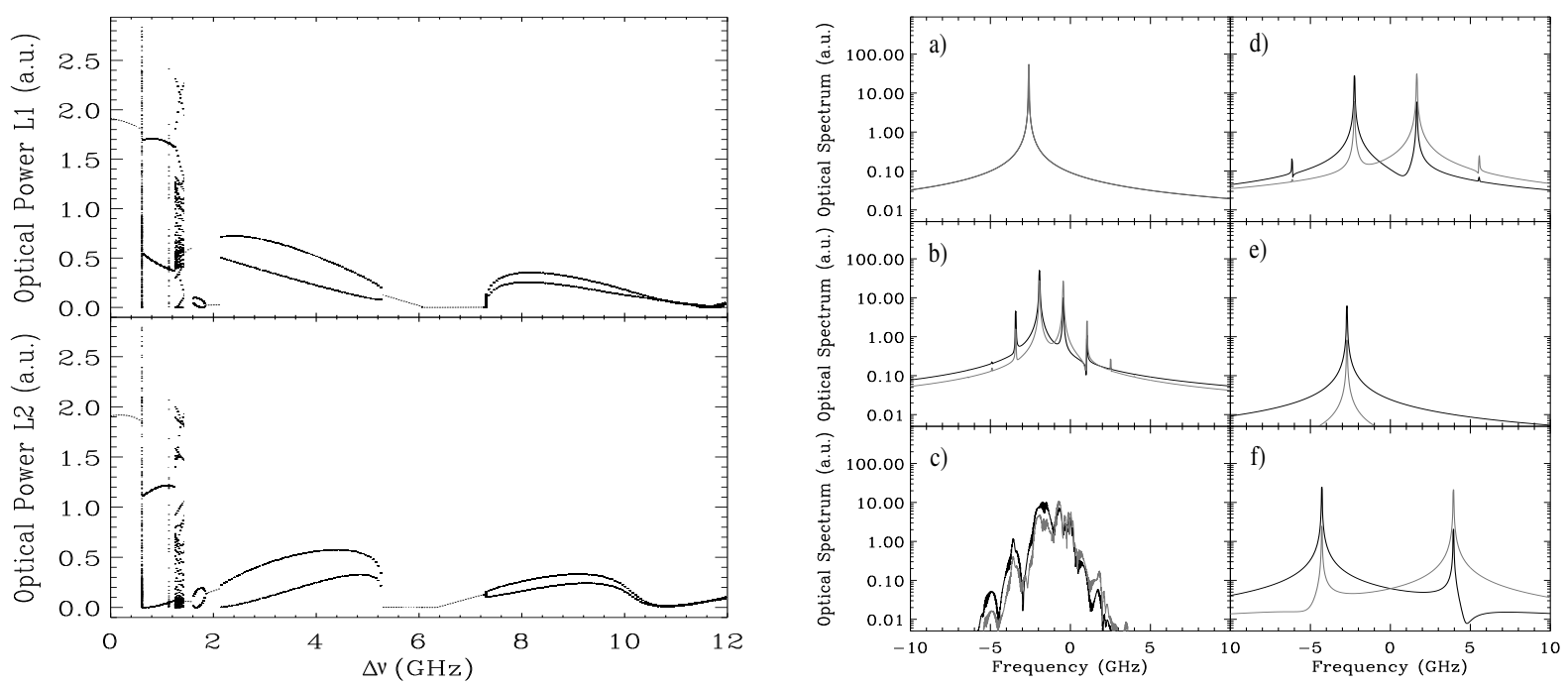

Figure 5. Left panel contains the bifurcation diagrams for the optical power of laser 1 and 2 against the detuning. Only extrema values are recorded. Right panel illustrates the optical spectra for: a) $\Delta \nu=0.4 \mathrm{GHz}, \mathrm{b}) \Delta \nu=0.8 \mathrm{GHz}, \mathrm{c}$ ) $\Delta \nu=1.3 \mathrm{GHz}, \mathrm{d}) \Delta \nu=4 \mathrm{GHz}$, e) $\Delta \nu=6 \mathrm{GHz}$, and f) $\Delta \nu=8 \mathrm{GHz}$. Black and grey colors distinguish the laser $1,2$.

d) both lasers are unlocked and oscillate around separated fixed points located approximately at $\approx \pm \Delta / 2$, e) the lasers lock again near the negative frequency state and f) the system comes back to an unlocked regime oscillating around different fixed points. It is worth noting that for the range of moderate values of detuning we have studied here $(\Delta<12 \mathrm{GHz})$, the number of steady-states decreases with the detuning until only two fixed points appear with frequencies close to $\approx \pm \Delta / 2$.

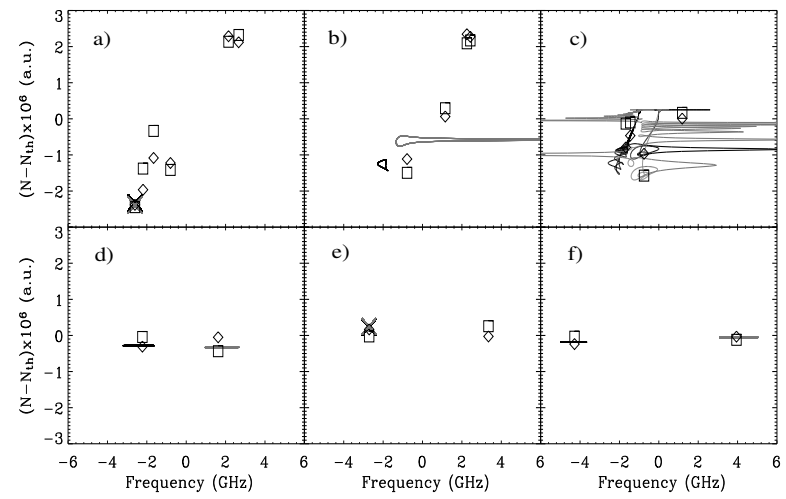

Figure 6. Fixed points and trajectories in the Inversion-Frequency plane for: a) $\Delta=0.4 \mathrm{GHz}, \mathrm{b}) \Delta=0.8 \mathrm{GHz}, \mathrm{c}$ ) $\Delta=1.3 \mathrm{GHz}$, d) $\Delta=4 \mathrm{GHz}$, e) $\Delta=6 \mathrm{GHz}$, and f) $\Delta=8 \mathrm{GHz}$. Diamonds and squares stand for the asymmetric fixed points of the laser 1 and 2, respectively. Crosses are used to identify the final states for the trajectories that reach a fixed point. Black and grey colors distinguish the laser 1 and 2 .

In all the previous studies, the propagation phase has been fixed to zero, despite it may play an important role in the dynamics. Now, we fix the detuning and change the phase in order to investigate the dynamical dependence on this critical parameter. Figure 7 contains the bifurcation diagram of both lasers when varying 
the phase for $0 \mathrm{GHz}$ (top panel) and $6 \mathrm{GHz}$ (bottom panel). In the non-detuned case, depending on the phase value chosen one can observe constant, periodic or even chaotic oscillations but always there exists a high degree of anticorrelation between the two laser outputs as it is shown in Figure 8. For larger detunings, the phase is only able to switch from stable to periodic behavior but no chaotic states can be achieved.
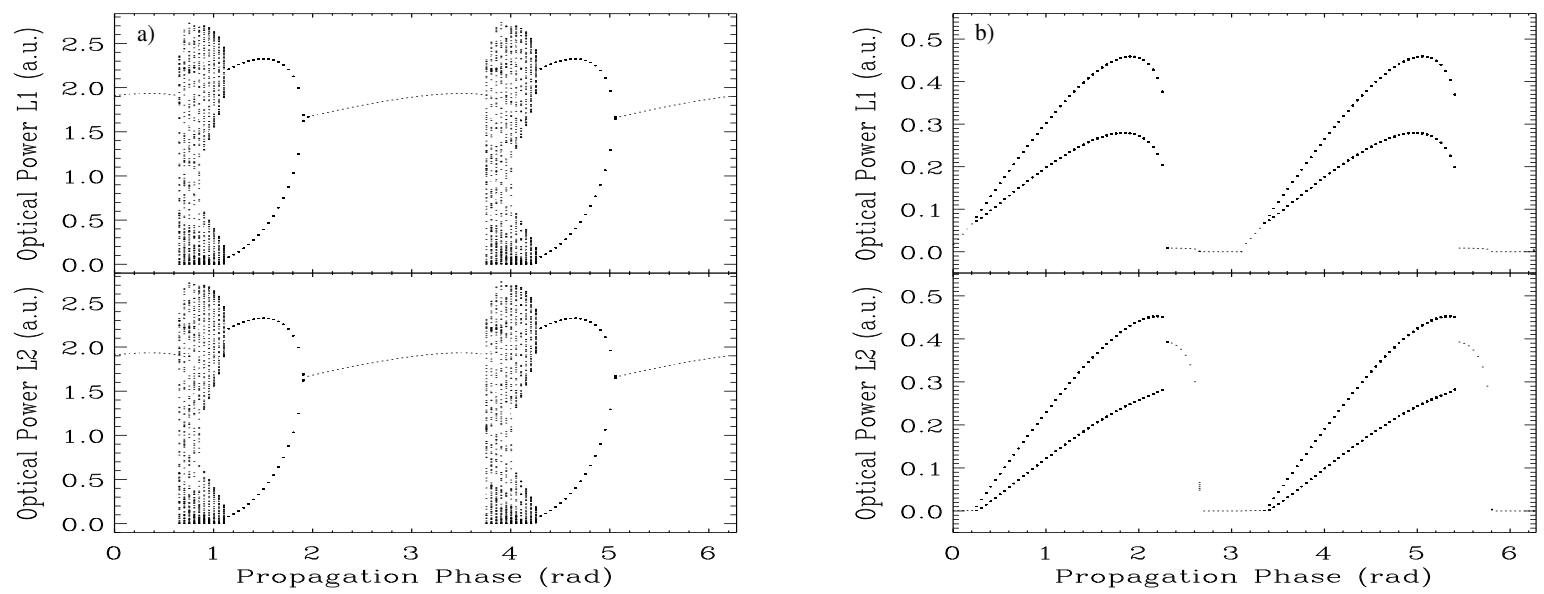

Figure 7. Bifurcation diagrams for the optical power of laser 1 and 2 against $\varphi_{0}$. a) $\Delta=0 \mathrm{GHz}$. b) $\Delta=6 \mathrm{GHz}$.
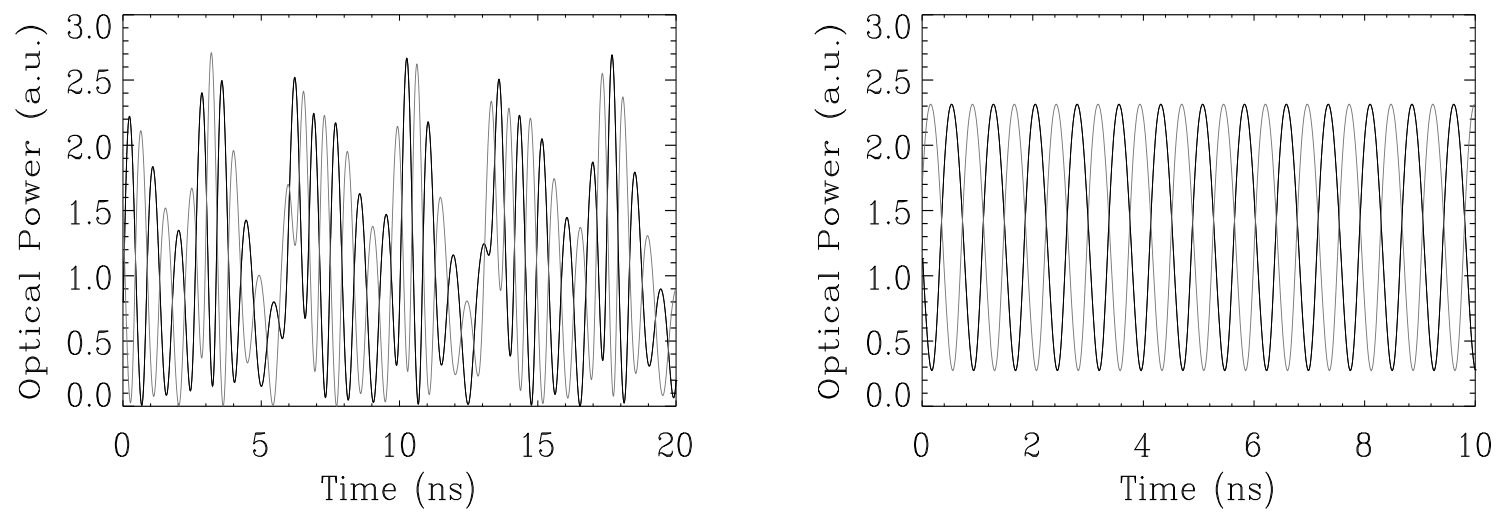

Figure 8. Temporal traces for the laser 1 and 2 showing anticorrelated dynamics for the non-detuned case. Left panel: $\varphi_{0}=0.8$ rad. Right panel: $\varphi_{0}=1.4 \mathrm{rad}$. Black and grey colors distinguish the laser 1 and 2 .

Now, we move to the long intercavity regime by increasing the distance between lasers up to $L=120 \mathrm{~cm}$ $(\tau=4 \mathrm{~ns})$. Here, the role of the phase $\varphi_{0}$ is not so important as in the short delay time case. The rest of the coupling conditions are the same than those used in the study of the short injection delay time. However, for this situation both lasers are in the LFF regime, at least for the non-detuned case. We gradually increase the solitary frequency of the laser 2 with respect to the laser 1, and we analyze the modification of the steady-states. We find very different situations: solutions with nearly the same inversion for the two lasers giving rise to almost the same optical power and solutions with very asymmetric operation of the two lasers. For small detunings, $\Delta \nu=0.5 \mathrm{GHz}$ (see Figure $9(\mathrm{a})$ ) we observe two slightly distorted ellipses with a maximum deformation close to their edges. The separation between the upper and lower branch of the ellipses decreases for the lower (higher) inversion modes of the low (high) frequency laser. For a larger detuning, $\Delta \nu=1.75 \mathrm{GHz}$ (see Figure 9(b)) we 
observe that the two ellipses split, giving rise to two new banana-like-shaped ellipses with very different inversion levels. In this case, and depending on the initial conditions, one can have operation in different regions of the diagram. Increasing further the detuning, the number of steady-states decreases until the boundary for the existence of frequency locked solutions is reached.

For a fixed detuning, we have analyzed the modification in the distribution of steady states when the injection current is increased observing that the metamorphosis of steady-states become more and more complex. Even for small detuning $(\Delta \nu=0.5 \mathrm{GHz})$, increasing the pumping level we observe that both ellipses split in two new ellipses with different inversion levels.

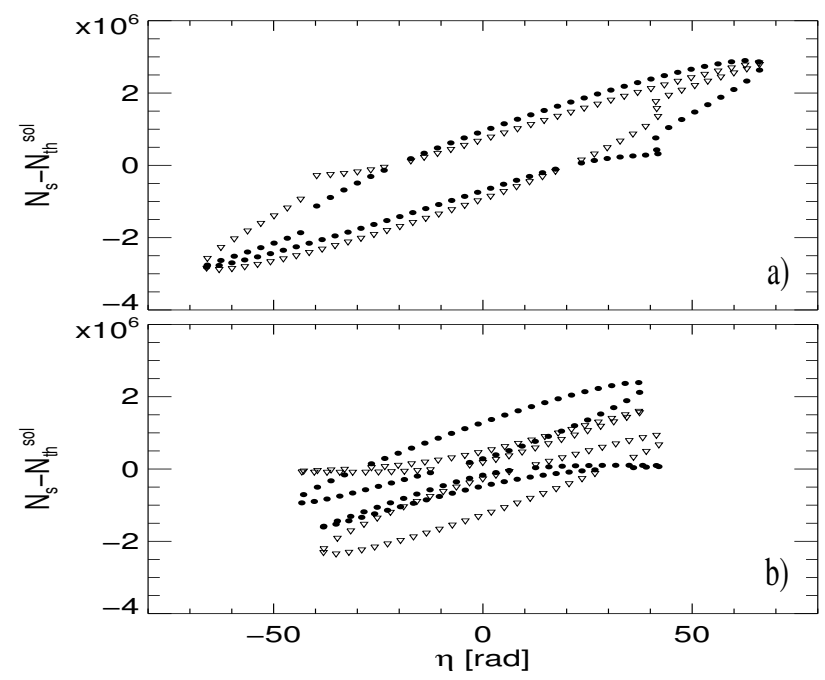

Figure 9. Steady-states in the Inversion-Frequency plane. Laser 2 (1) fixed points are represented by triangles (circles). a) $\Delta \nu=0.5 \mathrm{GHz}$. b) $\Delta \nu=1.75 \mathrm{GHz}$.

Regarding the temporal evolution of the system, we observed that for the zero-detuning case, the laser that drop first in the LFF regime seems to be randomly chosen, but as we discuss below this process becomes more regularized by increasing the detuning. Typical time traces of the intensity for different detunings are shown in Figure 10. Panel a) corresponds to $\Delta \nu=1 \mathrm{GHz}$. We observe power dropouts similar to those obtained in the resonant case but now appearing in a well defined leader-laggard sequence. The vertical lines in the figure indicate that the dropout of the higher frequency laser is followed by the dropout of the lower frequency one. The power dropouts become more periodic for $\Delta \nu=6 \mathrm{GHz}$ as shown in the panel b). We note that, in this case, the shape of the power dropouts differs from the usually one obtained under resonant conditions. The time traces for the lower frequency laser resemble to a sawtooth signal, while for the higher frequency laser resemble to a square one. For larger detuning, $\Delta \nu=8 \mathrm{GHz}$, in panel c), the time traces consist of dominant high frequency pulses of the intensity displaying some degree of correlation between the two series. For detuning larger than 12 $\mathrm{GHz}$ the synchronization is lost.

\section{CONCLUSIONS}

We have derived a potential picture of the phase dynamics of two semiconductor lasers coupled in a face to face configuration. Numerical comparison between this reduced and the full model shows excellent agreement in the low coupling strength limit. From this potential framework, the effect of the spontaneous emission noise and detuning are clearly identified. In particular, mode hopping characteristics and locking borders have been 


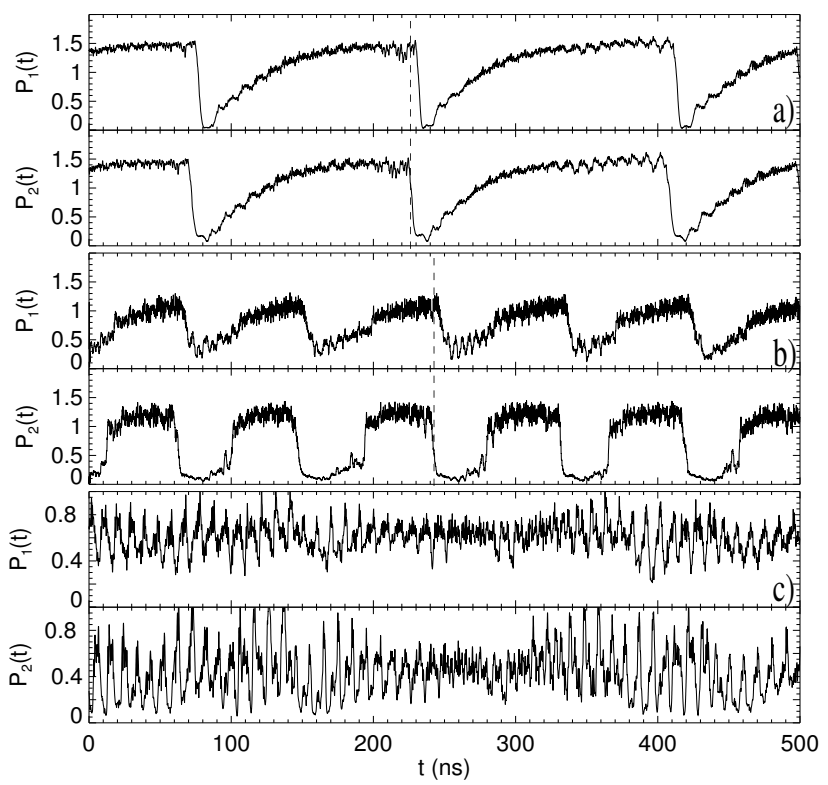

Figure 10. Typical time traces of the laser intensities under detuned operation. a) $\Delta \nu=1 \mathrm{GHz}, \mathrm{b}) \Delta \nu=6 \mathrm{GHz}$, and c) $\Delta \nu=8 \mathrm{GHz}$

predicted. Focusing on the short injection coupling time, the detuning-induced dynamics has been observed to pass through different stages. Interestingly, several islands of stable locking have been found to be located between regions of unlocking behavior. The role of the propagation phase have been also found to be critical in the short distance limit. Finally, in the long coupling delay time limit, we have studied how the LFF oscillations are modified in the presence of detuning. Rigorous study of the stability of the fixed points and limit cycles in the short intercavity regime, as well as a complete study of the relative dynamics between the lasers when parameters like the coupling strength or the coupling delay time are varied, are interesting subjects for future works.

\section{APPENDIX A}

The conditions to be imposed in order to obtain frequency-locked solutions are $E_{1}(t)=\sqrt{P_{1}} e^{i \Omega t}$ and $E_{2}(t)=$ $\sqrt{P_{2}} e^{i(\Omega t+\phi)}$ allowing a relative phase $\phi$ between the two fields. Furthermore, $\dot{P}_{1}=\dot{P}_{2}=0$ and $\dot{N}_{1}=\dot{N}_{2}=0$ in a steady-state. Introducing these conditions into eqs. (1-2) and neglecting Langevin noise sources, we arrive to a set of non-linear equations that reads

$$
\begin{aligned}
\eta+\delta & =-a C \sin \left(\eta+\varphi_{0}+\arctan \alpha-\phi\right), \\
\eta-\delta & =-\frac{1}{a} C \sin \left(\eta+\varphi_{0}+\arctan \alpha-\phi\right), \\
0 & =J_{k}-\gamma_{e} N_{k}-G_{k} P_{k} \quad k=1,2 .
\end{aligned}
$$

At this point, we have defined the following quantities: the compound system mode frequency $\eta=\Omega \tau$, the power ratio $a^{2}=P_{2} / P_{1}$, the normalized detuning $\delta=\Delta \tau$, the injection phase $\varphi_{0}=\Omega_{0} \tau$ and the effective coupling parameter $C=\kappa \tau \sqrt{1+\alpha^{2}}$. A solution of the system of equations (A1-A3) determines a vector of six unknowns $\left(P_{1}, P_{2}, N_{1}, N_{2}, \eta, \phi\right)$ that defines a frequency locked solution of the coupled system. Multiplying eq. (A1) by (A2) we find an expression for $y \equiv \sin (\phi)$, 


$$
y(\eta)= \pm \sqrt{\sin ^{2}\left(\eta+\varphi_{0}+\arctan \alpha\right)-\left(\eta^{2}-\delta^{2}\right) / C^{2}}
$$

while dividing eq. (A1) by (A2) we can obtain an expression for $a(\eta, y)$ that reads

$$
a^{2}=\left[\frac{\eta+\delta}{\eta-\delta}\right]\left[\frac{\sqrt{1-y^{2}}+y \cot \left(\eta+\varphi_{0}+\arctan \alpha\right)}{\sqrt{1-y^{2}}-y \cot \left(\eta+\varphi_{0}+\arctan \alpha\right)}\right]
$$

Finally, extracting from eqs. (A3) the power ratio $a^{2}$ and combining with eqs. (A4) and (A5), we arrive at the following non-linear equation for the compound system mode $\eta$

$$
a^{2}\left[\rho_{1}+a T_{+}\right]\left[a-b T_{-}\right]-\left[a \rho_{2}+T_{-}\right]\left[1-a b T_{+}\right]=0
$$

with $T_{ \pm}=\sqrt{1-y^{2}} \cos \left(\eta+\varphi_{0}\right) \pm y \sin \left(\eta-\varphi_{0}\right)$. In order to simplify notation we have introduced two additional parameters, $b=2 \kappa / \gamma$ and $\rho_{1,2}=\frac{g}{2 \kappa \gamma_{e}}\left(p_{1,2}-1\right) J_{t h}^{\text {sol }}$.

After solving eq. (A6) for $\eta_{s}$ and having avoided spurious solutions, we can use eqs. (A4) and (A5) to determinate the remaining unknowns $y_{s}, a_{s}$ and the following quantities

$$
\begin{aligned}
\phi & =\arcsin \left(y_{s}\right), \\
G_{1,2} & =\gamma-2 \kappa a_{s}^{ \pm 1} \cos \left(\eta_{s}+\varphi_{0} \mp \phi\right), \\
P_{1,2} & =\frac{\left(p_{1,2}-1\right) J_{t h}^{s o l}-\left(\gamma_{e} / g\right)\left(G_{1,2}-\gamma\right)}{G_{1,2}\left(1+s \gamma_{2} / g\right)}, \\
N_{1,2}-N_{t h}^{\text {sol }} & =\frac{1}{g}\left[G_{1,2}-\gamma\right]+s G_{1,2} P_{1,2} / g .
\end{aligned}
$$

\section{ACKNOWLEDGMENTS}

R.V., J.M. and C.R.M. acknowledge financial support from the Ministerio de Ciencia y Tecnología (Spain) and FEDER and BFM2002-04369) and from the EC project IST-2000-29683 OCCULT. C.R.M. is also grateful for the hospitality and support of the Electrical Engineering Department, University of California, Los Angeles. C.R.M is also funded by the Secretaría de Estado de Educación y Universidades, Ministerio de Educación, Cultura y Deporte, Spain. Marc Sciamanna is a Research Fellow from the Fonds National de la Recherche Scientifique (FNRS), Belgium.

\section{REFERENCES}

1. J. Mork and B. Tromborg, "The mechanism of mode selection for an external cavity laser," IEEE Phot. Tech. Lett. 2, p. 21, 1990.

2. J. Mork, M. Semkow, and B. Tromborg, "Measurement and theory of mode hopping in external cavity lasers," Electronics Lett. 26, p. 609, 1990.

3. D. Lenstra, "Statistical theory of the multistable external-feedback laser," Optics Comm. 81, p. $209,1991$.

4. A. Glova, S. Kurchatov, V. Likhanskii, A. Lysikov, and A. Napartovich, "Coherent emission of a linear array of $\mathrm{CO}_{2}$ waveguide lasers with a spatial filter," Quant. Electron. 26, p. 500, 1996.

5. H. Haken, J. Kelso, and H. Bunz, "A theoretical model of phase transitions in human hand movements," Biol. Cybern. 51, p. 347, 1985. 
6. A. Pikovsky, M. Rosenblum, and J. Kurths, Synchronization: A Universal Concept Nonlinear Science, Cambridge University Press, 2002.

7. A. Hohl, A. Gavrielides, and V. Erneux, T. Kovanis, "Localized synchronization in two coupled nonidentical semiconductor lasers," Phys. Rev. Lett. 78, p. 4745, 1997.

8. H. T., I. Fischer, W. Elssser, J. Mulet, and C. Mirasso, "Chaos synchronization ans spontaneous symmetrybreaking in symmetrically delay-coupled semiconductor lasers," Phys. Rev. Lett. 86, p. 795, 2001.

9. J. Mulet, C. Masoller, and C. R. Mirasso, "Modeling bidirectionally coupled single-mode semiconductor lasers," Phys. Rev. A 65, p. 063815, 2002.

10. J. M. Buldu, R. Vicente, T. Perez, C. R. Mirasso, M. C. Torrent, and J. Garca-Ojalvo, "Periodic entrainment of power dropouts in mutually coupled semiconductor lasers," Appl. Phys, Lett. 81, p. 5105, 2002.

11. J. Javaloyes, P. Mandel, and D. Pieroux, "Dynamical properties of lasers coupled face to face," Phys. Rev. E 67, p. 036201, 2003.

12. R. Vicente, J. Mulet, C. R. Mirasso, S. Tang, and J. Liu, "Dynamical properties of two semiconductor lasers with bidirectional optoelectronic coupling," Physics and Simulation of Optoelectronic Devices XI, SPIE Proceedings 4986, p. 440, 2003.

13. F. Rogister, A. Locquet, D. Pieroux, M. Sciamanna, O. Deparis, P. Megret, and M. Blondel, "Secure communication scheme using chaotic laser diodes subject to incoherent optical feedback and incoherent optical injection," Optics Letters 26, p. 1486, 2001.

14. A. Hohl, A. Gavrielides, and V. Erneux, T. Kovanis, "Quasiperiodic synchronization for two delay-coupled semiconductor lasers," Phys. Rev. A 59, p. 3941, 1999.

15. R. Lang and K. Kobayashi, "External optical feedback effects on semiconductor injection laser properties," IEEE J. of Quantum Electron. 16, p. 347, 1980.

16. T. Saaty, Modern Nonlinear Equations, Dover, 1981.

17. J. Mulet, C. Mirasso, T. Heil, and I. Fischer, "Synchronization scenario of two distant mutually coupled semiconductor lasers," J. Opt. B: Quantum Semiclass. Opt. 6, p. 97, 2004.

18. P. Hanggi, P. Talkner, and M. Borkovec, "Reaction-rate theory: Fifty years after kramers," Rev. Mod. Phys. 62, p. 251, 1990.

19. G. Tartwijk and D. Lenstra, "Semiconductor lasers with optical injection and feedback," J. Opt. B: Quantum Semiclass. Opt. 7, p. 87, 1995. 